\title{
Measuring Blood Pressure in Clinical Practice: We Can and Must Do Better
}

Therapeutic Innovation \& Regulatory Science 2018, Vol. 52(6) 685-686 (C) DIA 2018

Article reuse guidelines: sagepub.com/journals-permissions DOI: 10.1 177/2168479018809978 tirs.sagepub.com
Many readers (at least those living in the United States who are blessed with comprehensive health insurance and those living in a country where access to good health care is a fundamental right) are used to having your blood pressure measured when visiting a doctor's office, whether for a particular concern or for an annual physical examination. Blood pressure measurement is an important aspect of evaluating general health and is often employed as an initial diagnostic tool. It is also measured when treating a patient for high blood pressure (hypertension). However, despite this ubiquity, a very strong word of caution is appropriate: it is remarkably (and scarily) easy to obtain $a$ blood pressure reading, but much more difficult to obtain an accurate blood pressure reading at that particular point in time. ${ }^{1}$ Moreover, as discussed in due course, even an accurate single reading can be considerably misleading.

Before getting into further detail, it is appropriate to consider how blood pressure measurements have historically been taken, and how the unit of measurement, millimeters of mercury ( $\mathrm{mmHg}$ ) arose. If channeled to the bottom of a column of mercury, the pressure in the main artery leaving the heart would cause the mercury to rise to a certain number of millimeters in height: higher pressure would result in a greater rise. The first (noninvasive) measures of blood pressure employed a mercury column that rose and fell during the cardiac cycle. You may wonder why mercury - a chemical now known to cause brain injury and lung injury - was chosen. The reason is that mercury is approximately 13 times heavier than water. Therefore, had a column of water been employed, it would have had to be 13 times higher, which essentially means that it would have gone through the ceiling in most doctors' offices. Such sphygmomanometers are not typically used nowadays, but the nomenclature remains.

Getting back now to the main narrative of how a better job of assessing blood pressure can and must be done, consider a scenario involving going to your doctor's office for a routine physical examination at a time of the year when it is cold. It is possible, given your busy schedule, that you are running tight on time and therefore perhaps driving a bit faster (and, therefore, experiencing more stressful driving circumstances than usual) from wherever you start the trip to the parking lot at or near your doctor's office. You then walk briskly for several minutes to get to the office. Since you are running tight on time, the nurse or physician's assistant is waiting for you and summarily takes a single blood pressure reading, quite possibly while you are wearing a long-sleeved shirt or blouse and a wool sweater. In this case, it is quite possible (even likely) that the blood pressure cuff will be placed around these items of clothing, not next to your upper arm skin.

For several reasons, the resulting blood pressure reading that is documented in your health records probabilistically does not bear any semblance of accuracy. First, you should be allowed to rest for a certain period of time before readings (pluralmore shortly) are taken. Second, placing the blood pressure cuff on top of various layers of clothing is not optimal. Third, the health care practitioner may have quickly inflated and far too quickly deflated the blood pressure cuff. Fourth, a single measurement under any circumstances invites inaccuracy that can be avoided by a series of readings of which the first can be discarded and the subsequent ones averaged.

Let me take a brief departure from clinical blood pressure assessments to assessments made by our veterinarian for one of our cats. ${ }^{2}$ During a routine physical examination, she had her blood pressure taken in a laboratory while my wife and I waited in a separate room. In due course, the vet presented us with a sheet of paper on which multiple values were meticulously recorded, and then discussed them with us. Five sequential assessments of systolic blood pressure and the accompanying diastolic blood pressure had been made. For both blood pressure parameters, a pattern that will be familiar to clinicians who actually take multiple readings from human patients was readily apparent. The fifth systolic and diastolic values were each considerably lower than the respective first values, and the descent from first to fifth values was close to linear in each case. This rigorous assessment protocol allowed the vet to make an optimally informed blood pressure assessment (as it happens, an antihypertensive regimen was initiated).

Two points are noteworthy here. First, the late, great Dr Tom Pickering was instrumental in the term "white coat hypertension" becoming widely used in the scientific literature and clinical practice. The fundamental notion is that many humans experience a certain degree of apprehension when going to a physician's office — physicians typically wear white coats - and blood pressure can therefore be elevated above normal levels by this experience. While our vet did not use the term white coat hypertension in his discussions with us, he was clearly cognizant of the ramifications of this phenomenon for animals, hence the nature of his rigorous blood pressure assessment protocol. Second, the point of this Editorial is certainly not to criticize individual physicians, but rather to lament the current state of clinical practice in countries in which physicians feel that the time necessary for the implementation of such a rigorous protocol is not afforded to them by the bean 
counters that control the finances of health care. Therefore, the conduct of the desirable level of rigor can often be "shrouded by impracticality." ${ }^{2}$ My hope is that this situation will change for the better in the future.

In addition to manual readings taken using a stethoscope and sphygmomanometer, several other modalities permit blood pressure assessment in clinical practice. These include automated office blood pressure measurement, home blood pressure measurement, and ambulatory blood pressure monitoring $(\mathrm{ABPM})^{3}$ : this reference is available at no cost on PubMed. As Pickering and colleagues ${ }^{4}$ observed, "Any clinical measurement of blood pressure may be regarded as a surrogate measure for the 'true' blood pressure of the patient, which may be defined as the mean level over prolonged periods." ABPM provides multiple readings across a 24-hour period, facilitating assessment of mean 24-hour, daytime, and nighttime values, thus better representing "true" values as well as variability within these means. In addition to proving informative in the context of investigating the efficacy of antihypertensive agents, ${ }^{5}$ current evidence "now overwhelmingly shows that ambulatory blood pressure monitoring is mandatory for the proper management of hypertension." 6

I would like to refer readers to the July 2018 issue of the Journal of Clinical Hypertension, a special issue titled "Blood Pressure Measurement: A Reappraisal for 21st Century Practice." Edited by Dr Eoin O'Brien and Dr George Stergiou, this volume is devoted entirely to state-of-the-art recommendations on the measurement of office, ambulatory, and home blood pressures. The issue's 13 papers are written by 27 authors from various countries who are all authorities in their field, and the papers are available at no cost on PubMed. The purpose of the issue is to provide "a forum in which to summarize the current status of blood pressure measurement and, most importantly, to lay down recommendations for the future." I I encourage you to read these excellent papers, and then, should you wish, have a conversation with your doctor about his or her assessment of your blood pressure.

$$
\begin{array}{r}
\text { J. Rick Turner, PhD, DSc, FASH, FACC, FESC, FCP } \\
\text { Editor-in-Chief } \\
\text { Therapeutic Innovation \& Regulatory Science }
\end{array}
$$

\section{References}

1. Turner JR. New Drug Development: An Introduction to Clinical Trials. 2nd ed. New York: Springer; 2010.

2. Turner JR. Letter to the Editor. Can clinical practice learn about measuring blood pressure from our veterinary colleagues? J Clin Hypertension (Greenwich). 2017;12:1385.

3. Kindman LA, Turner JR, Lee J. Blood pressure measurement modalities: a primer for busy practitioners. J Clin Hypertens (Greenwich). 2016;18:262-266.

4. Pickering T, Shimbo D, Haas D. Ambulatory blood-pressure monitoring. N Engl J Med. 2006;354:2368-2374.

5. Stockbridge S. Letter to the editor: ABPM part of FDA reviews. Drug Inf J. 2011;45:567.

6. Staessen JA, Li Y, Hara A, et al. Blood pressure measurement anno 2016. Am J Hypertens. 2017;30:453-463.

7. O'Brien E, Dolan E, Stergiou GS. Achieving reliable blood pressure measurements in clinical practice: It's time to meet the challenge. J Clin Hypertens (Greenwich). 2018;20:1084-1088. 